\section{SACRAL NERVE NEUROMODULATION THERAPY; A PROMISING NEW TREATMENT FOR CHILDREN WITH REFRACTORY FUNCTIONAL CONSTIPATION}

B. Peeters ${ }^{1}$, B.P.W. van Wunnik², W. van Gemert ${ }^{2}$, M.A. Benninga ${ }^{1}$, C.G.M.I. Baeten ${ }^{2}$

${ }^{1}$ Department of Pediatric Gastroenterology and Nutrition, Emma Children's Hospital, Academic Medical Center Amsterdam, Amsterdam, ${ }^{2}$ Department of Surgery, University Medical Center Maastricht, Maastricht, The Netherlands

Background: Sacral nerve neuromodulation (SNM) therapy has been successfully applied in adult patients with urinary and fecal incontinence. Recently, SNM therapy showed good results in adults with refractory functional constipation. No data, however, are available of SNM therapy as a treatment option in children with refractory functional constipation.

Aim: To describe the preliminary results of SNM therapy in children with chronic functional constipation refractory to intensive conservative treatment.

Material and methods: Children with functional constipation according to the ROME III criteria not responding to intensive conservative treatment (laxatives, enemas and colonic lavage) were assigned for SNM therapy. When improvement of symptoms was seen during the percutaneous nerve evaluation (PNE) with a permanent electrode in the 3 weeks testing phase, a permanent sacral nerve neuromodulator was implanted. Patients were followed up for at least six months after implantation by interviews and bowel diaries. Improvement was defined as spontaneous defecation $\geq 3$ times a week.

Results: 5 patients (all females) 14 - 18 years old with functional constipation were included. None of them had spontaneous defecation at presentation. Duration of symptoms varied from 6 - 18 years and the duration of treatment from 1-17 years. In all patients the testing phase was successful. After implantation of the permanent neuromodulator, all children had spontaneous defecation $\geq 3$ times a week without medication.

Conclusion: SNM is a promising new treatment option in children with refractory functional constipation notresponding to intensive conservative therapy. However, larger prospective studies with long-term follow up are required.

270

\section{PORTAL VEIN THROMBOSIS IN NEONATES WITH UMBILICAL VASCULAR CATHETERIZATION}

M.M. Gharehbaghi ${ }^{1}$, A. Peirovifar ${ }^{2}$, M. Nemati ${ }^{2}$, S. Hossain Pour ${ }^{2}$

${ }^{1}$ NICU, ${ }^{2}$ Tabriz University of Medical Sciences, Tabriz, Iran

Background: Umbilical vascular catheters are routinely inserted in critically ill newborn infants in most neonatal intensive care units (NICU). The objectives of this study were to determine catheter-associated thrombosis by color Doppler ultrasonography and to detect duration of catheter placement as a risk factor for thrombosis.

Material and methods: All newborn infants with umbilical vascular catheterization for more than $6 \mathrm{~h}$ duration were included in this study. Color Doppler ultrasonography was performed within24-48 hours of catheter insertion, 48-72 $\mathrm{h}$ after withdrawal and at weekly intervals until hospital discharge or clot resolution.

Results: Ninety five (58\%) of patients were boys. The most common underling medical condition in studied patients were respiratory distress syndrome Portal vein thrombosis (PVT) was determined in 5 cases (\%3.04) of 164 infants received umbilical vascular catheterization. The mean duration of

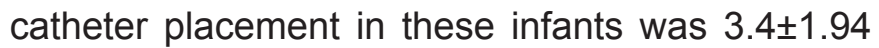
day, which was not significantly different from infants without thrombosis $(3.5 \pm 2.03)$. Thrombosis completely recanalized and resolved after 3-6 weeks in three survived neonates. There was history of exchange transfusion for hyperbilirubinemia via umbilical vein in two neonates with PVT.

Conclusion: Catheter-associated portal venous thrombosis was uncommon in our study. The duration of catheter placement was not longer in patients with portal vein thrombosis than those without thrombosis. 